\title{
Emulsified lipids: formulation and control of end-use properties
}

\author{
Fernando LEAL-CALDERON \\ Laboratoire de chimie et biologie des \\ membranes et des nanoobjets \\ (CBMN, UMR CNRS 5248), \\ Université Bordeaux 1, \\ Allée Geoffroy Saint-Hilaire, \\ 33600 Pessac, France \\ $<$ fleal@enscbp.fr $>$
}

\begin{abstract}
In many practical applications including foods, cosmetics, pharmaceuticals, etc., lipids are emulsified in an aqueous phase in the presence of surface-active molecules and other additives like thickening/gelling agents. Once fabricated, the emulsions may exhibit all kinds of rheological behaviors from viscous fluid to elastic pastes, and transitions: reversible phase transitions as a result of droplet interactions that may be modified to a large extent, and irreversible transitions that generally involve their destruction. Besides the predominance of empiricism in controlling most of the end-use properties, the scientific background of emulsions is progressing. In this paper we aim to review some advances concerning the control of the structure, the texture (rheological properties) and the ageing of emulsions.
\end{abstract}

Key words: emulsions, lipids, triglycerides, surfactants, proteins, fat crystals, coalescence, Ostwald ripening, flocculation, gelling, shear

(called "interface") at the droplets surface. Molecules made of a linear hydrocarbon chains (between 10 and 20 methyl segments) and a polar head group, commonly named surfactants, fall into this class of molecules. Biopolymers like proteins or some polysaccharides may also be used as surface-active species. Their functional role in emulsions is crucial since (i) they facilitate the emulsification process, (ii) they prolong the shelf life of the emulsions (see below) and (iii) they determine the emulsion type $(\mathrm{O} / \mathrm{W}$ or $\mathrm{W} / \mathrm{O})$ according the well known Bancroft rule ("the phase in which the surfactant is preferentially soluble tends to become the continuous

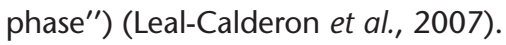

The surface tension $(\sigma)$ is a measure of the cohesive energy arising from the imbalance of forces between the molecules at an interface. When two immiscible phases are in contact with each other, then the molecules at the interface experience an imbalance of forces. This will result in an increase of the free energy at the interface. This excess free energy can be quantified as the amount of energy required to create a new surface or as the force that acts perpen- dicular and inward from the boundaries of the surface to reduce the area of the interface. The unit of interfacial tension in the $\mathrm{SI}$ system is $\mathrm{N} \cdot \mathrm{m}^{-1}$. From a thermodynamic point of view, emulsions are out-of-equilibrium (metastable) systems because of surface tension. As such, they tend to evolve over time towards the total phase separation of the two immiscible phases. As a matter of fact, the kinetic stability of emulsions is strongly dependent on its formulation, and especially on the type and concentration of the dispersed phase and of the surface-active species. Because the shelf life of these materials may become significant (from weeks to years) they are suitable for various commercial applications.

Emulsions of the $\mathrm{O} / \mathrm{W}$ type are widely used because of their ability to safely transport and deliver hydrophobic substances through an aqueous continuous phase. All kinds of surface treatments are based on emulsion technology: painting, paper coating, road surfacing, etc. Because homogeneous mixtures of two immiscible fluids may be obtained, hazardous solvents may be avoided when transporting hydrophobic sub- 
stances. Once the emulsion is spread, water evaporates and is released into the atmosphere, while the dispersed phase concentrates, leading to the formation of a hydrophobic film. Moreover, emulsion technology significantly simplifies the handling and pouring of many hydrophobic substances. Indeed, at ambient temperature some materials may be almost solid, whereas by dispersing them in water, they remain fluid. Typical examples are bitumen used for road surfacing or silicon oil used to ensure water-tightness in bathroom fittings. Emulsions are also involved in food and cosmetic industry because of the large variety of rheological properties and sensory profiles that may be achieved. Moreover, they are also efficient drug carriers (medicines, food, and pesticides) for various biological targets.

The ingredients of emulsions self-organize under the effect of physical (intermolecular or interdroplet forces, phase separations), chemical (formation of covalent bonds) and biological (fermentation, etc.) factors. Because emulsions are metastable systems, their initial microstructure evolves over time during storage and/or transportation as a result of thermal treatments, mechanical stresses, physical coarsening and biological action. An important challenge for emulsion science is therefore to understand and control the evolution of colloidal interactions, microstructure and rheological properties during processing, storage, transportation, application and even ingestion (food emulsions).

All the industrial applications have already led to an important empirical control of emulsions from their preparation to their destruction. The scientific background of emulsions has also progressed over the last decades and in this tutorial review we aim to give an overview of some recent advances, emphasizing the functionality of the ingredients, the control of the rheological properties through the formulation or upon application of a shear, and the coarsening (ageing) mechanisms.

\section{Overall composition and fabrication}

The behavior of emulsions is determined by the three phases of the system: the fat or oil phase, the interfacial phase and the aqueous phase. Understanding the structural properties of emulsions requires a precise knowledge of the behaviors of these three phases, both individually and cooperatively. In O/W emulsions based on lipids, the dispersed phase is mainly composed of triglycerides that may be partly or totally crystallized depending on the temperature. The aqueous phase may contain ions, biomolecules such as polysaccharides or proteins, etc. which may exert stabilizing or destabilizing effects.

The interface may be composed of proteins, amphiphilic polymers and/or small surfactants. The surface-active species that can be used is restricted by regulations, cost considerations and practical factors, such as ease of utilization reliability of source and compatibility with other ingredients. Common stabilizers used in the food, cosmetic and pharmaceutical industries to formulate emulsions are:

- amphiphilic proteins like whey protein isolates, caseinates, soy protein isolates;

- amphiphilic polysaccharides like modified starch, locust beam gum, gum Arabic, etc.;

- low-molecular weight surfactant molecules like "Spans" (sorbitan fatty acid esters), "Tweens" (ethoxylated sorbitan esters), fatty acids, mono- and di-glycerides;

- phospholipids like egg, soy or dairy lecithin.

Being metastable, emulsions are not formed spontaneously once the ingredients are put in contact. Instead, their manufacturing generally requires the application of an intense shear. A variety of methods have been developed to prepare emulsions which can be classified as either turbulent or laminar flow techniques. Turbulent flow techniques are based on mechanical devices generating turbulence and cavitation phenomena that allow breakup of the dispersed phase and lead to the formation of tiny droplets. Among these techniques, we can mention highpressure homogenizers, microfuidizers or turbulent blade-type mixers (LealCalderon et al., 2007). Laminar flow techniques rely on the application of low shear rates $\dot{\gamma}$ in highly viscous emulsions. In this regime, the applied stress $\tau=\eta \dot{\gamma}$, where $\eta$ is the emulsion viscosity, must be large enough to produce droplet elongation and further break-up according the so-called Rayleigh instability (Mabille et al., 2000). Droplet fragmentation is possible at low shear rates provided the average emulsion viscosity is large enough.

A conventional emulsion typically has droplets with mean radii between $100 \mathrm{~nm}$ and $100 \mu \mathrm{m}$. Conventional emulsions are optically opaque because the droplets have similar dimensions to the wavelength of light and so they scatter light strongly. A nanoemulsion is an emulsion that contains very small droplets, i.e. mean radii between 10 to $100 \mathrm{~nm}$. One of the main attractions of nanoemulsions over conventional emulsions is that they tend to be optically transparent. Consequently, they can be used to incorporate lipophilic bioactive molecules into transparent, aqueousbased products or beverages without altering too much their appearance. Nanoemulsion delivery systems may also protect the encapsulated bioactives against environmental stresses (e.g. pH, oxygen, and degradation by enzymes), and increase the bioavailability of the encapsulated bioactives because of their small droplet size and high surfaceto-volume ratio (McClements, 2011). Nanoemulsion-based delivery systems are well established in the pharmaceutical domain where they improve the oral bioavailability and biological efficacies of lipophilic drugs (Shah et al., 2010). Nanoemulsions are attracting much interest in the food and nutraceutical industries owing to their potential to incorporate and maximize the health benefits of lipophilic functional components within food products (e.g. $\varpi-3$ fatty acids, $\alpha$-tocopherol, lycopene, lutein, coenzyme Q10, etc.).

\section{Ageing: coalescence and Ostwald ripening}

Once formed, the shelf life of emulsions may vary from a few hours to years depending on formulation and process parameters. Figure 1 illustrates the main mechanisms of physical emulsion instability. Some instabilities involve droplet aggregation and are generally reversible; others, related to droplet size evolution, are irreversible (Leal-Calderon et al., 2007). Reversible flocculation may lead emulsions to cream (O/W emulsions) or sediment $(\mathrm{W} / \mathrm{O})$ depending on the overall density mismatch between the 


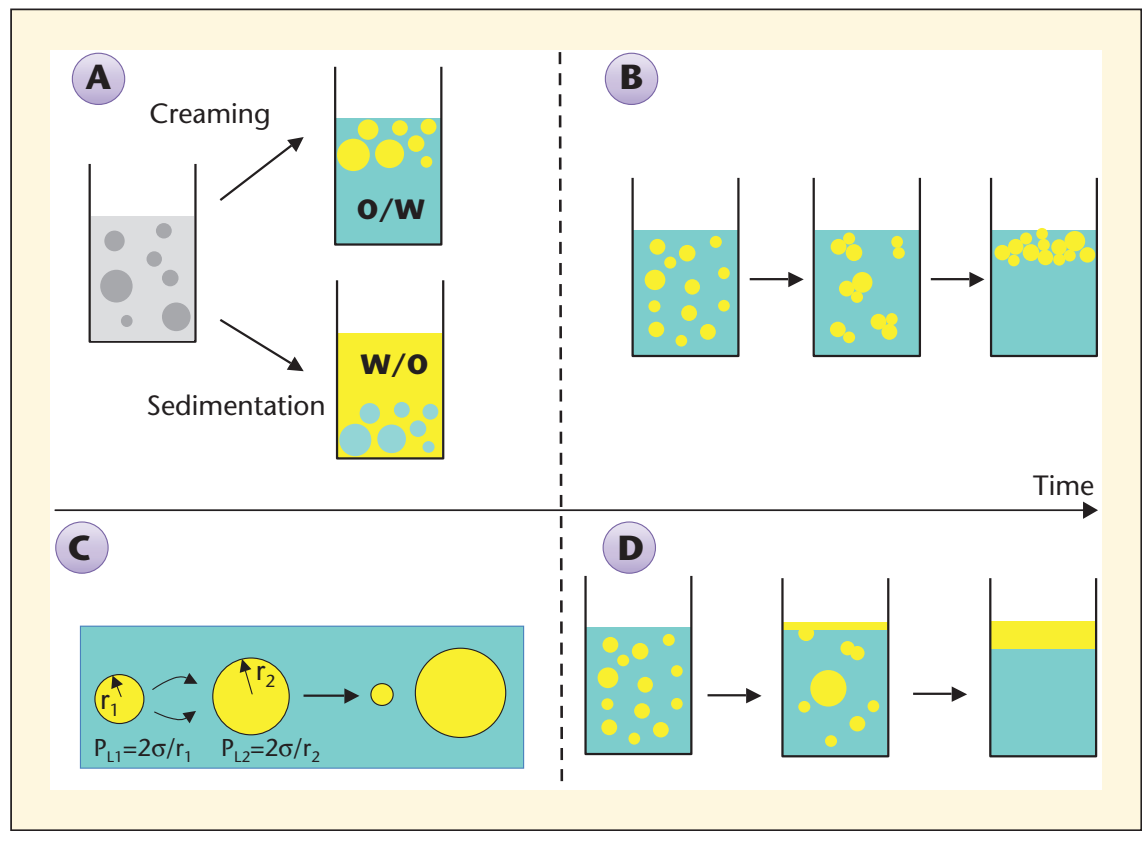

Figure 1. Schematic representation of the different instabilities in emulsions. A) creaming or sedimentation due to the density mismatch between the drops and the continuous phase; $B$ ) flocculation (aggregation) followed by creaming (O/W emulsions); C) Ostwald ripening (Diffusion of dispersed molecules from small droplets to large ones across the continuous phase, because of the difference in Laplace Pressure); D) coalescence (the drops recombine ultimately leading to the total phase separation of the two immiscible phases).

droplets and the continuous phase. Meanwhile, irreversible droplet size increase may occur through two different mechanisms: Ostwald ripening and coalescence.

If the average diameter in the emulsion is lower than approximately $1 \mu \mathrm{m}$, the droplets are submitted to thermal (Brownian) motion that delays gravity induced phase segregation phenomena. However, creaming or sedimentation is accelerated if the droplets undergo flocculation. For instance, high levels of salt are known to promote aggregation in emulsions by screening the repulsive interaction between the droplets. Above a critical salt concentration, the electrostatic repulsion is no longer sufficient to overcome the attractive van der Waals forces (Israelechvili, 1985). Protein-stabilized emulsions appear to be more stable to high salt levels than conventional emulsions because the presence of a thick protein layer induces a long-range steric repulsion (Israelachvili and Wennerström, 1992; Dimitrova et al., 2004). Two emulsions droplets may also attract each other when surrounded by smaller species. This attraction is purely entropic in origin: when the two large droplets approach, neous curvature, etc. (Kabalnov and Wennerström, 1996; Leal-Calderon et al., 2007). When coalescence is the main destabilization mechanism, the time evolution of the average droplet size is self-accelerated and the size distribution broadens (Schmitt et al., 2004).

Ostwald ripening (OR) consists of the net transport of oil from small droplets to larger ones through the continuous phase. OR in O/W emulsions occurs via the molecular dissolution of oil in the continuous phase. It is driven by the dependence of the oil solubility with droplet size:

$C(r)=C(\infty) \exp \left(\frac{2 \sigma V_{m}}{r R T}\right)$

where $C(r)$ is the solubility of the emulsified oil, $C(\infty)$ is the oil solubility close to a planar interface, $r$ is the droplet radius, $\sigma$ is the interfacial tension, $V_{m}$ is the oil molar volume and $\mathrm{R}$ is the ideal gas constant. Equivalently, this means that the chemical potential, $\mu(r)$, of the dispersed phase depends on the droplet radius, $r$ :

$\mu(r)=\mu(\infty)+\frac{2 \sigma V_{m}}{r}$

the small species may be expelled, leading to an uncompensated pressure within the depleted region. The so-called depletion attraction scales with the osmotic pressure of the small species and also with the size of the depleted volume in between the two large interacting droplets (Asakura and Oosawa, 1958). Various types of tiny colloidal species may produce depletion flocculation, when present in large excess in the continuous phase: polymer coils (Meller and Stavans, 1996), surfactant aggregates (micelles) (Aronson, 1989; Bibette et al., 1990), or proteins (Dickinson et al., 1997).

Coalescence occurs when two droplets collide and finally merge. At a microscopic scale, a single coalescence event between two drops in contact proceeds through the nucleation of a channel that reaches a critical size, above which it becomes unstable and grows. For a coalescence event to occur, an energy barrier has to be overcome (Kabalnov and Wennerström, 1996). Its origin is still a matter of debate but it is now well admitted that the activation energy may be determined by several interfacial parameters: surface forces, surface tension, dilatational elasticity, sponta- where $\mu(\infty)$ is the chemical potential at a planar interface. The second term in the rand-hand side of eq. 2 represents the energy due to the additional pressure within the droplets with respect to the continuous phase, also known as Laplace pressure $(2 \sigma / r)$. Thus $\mathrm{OR}$ arises from the difference in chemical potential between small and large emulsion droplets.

The theoretical models developed by Lifshitz-Slesov (1961) and Wagner (1961) predict a linear relationship between the cube of the average emulsion radius $\langle r\rangle$ and time, the slope of which is:

$\omega=\frac{d<r>^{3}}{d t}=\frac{8}{9}\left[\frac{C(\infty) \sigma V_{m} D}{\rho R T}\right]$

where $D$ is the molecular diffusion coefficient of the dispersed molecules in the continuous phase and $\rho$ is the density of the dispersed phase. The theory also predicts that the size distribution remains narrow and self-similar over time, that is the same distribution is obtained when plotted as a function of $\mathrm{r} /<\mathrm{r}>$.

OR can be slowed down by dissolving in the dispersed phase a second oil with 
much lower water solubility (Higuchi and Misra, 1962). The concept is based on the entropy of mixing that opposes OR (Kabanov, 2001). When a mixed oil emulsion undergoes $\mathrm{OR}$, the soluble oil has greater mobility. Over time, the larger droplets become enriched with the soluble oil whereas smaller droplets become enriched with the insoluble compound. This situation creates an osmotic pressure mismatch that tends to drive the soluble oil back to the smaller droplets, thus compensating the effect of OR. In an ideal mixture, the chemical potential of the soluble oil is given by:

$\mu(r)=\mu(\infty)+\frac{2 \sigma V_{m}}{r}+R T \operatorname{Ln}\left(1-x_{i}\right)$

where $x_{i}$ is the mole fraction of insoluble oil in the droplet. The Laplace pressure term in the rand-hand side of this equation is always positive. Compared with eq. 2, the additional term represents the entropy of mixing, which is negative for any fraction $x_{i}$. It can be deduced from eq. 4 that an emulsion can be stabilized against OR provided the fraction of the insoluble compound is large enough. According to Kabalnov's theoretical treatment (2001), the entropic stabilization is obtained when the osmotic pressure of the insoluble compound is comparable to Laplace pressure. The minimum amount of insoluble compound to be incorporated is thus:

$x_{i, \min } \sim \frac{2 \sigma V_{m}}{<r_{0}>R T}$

where $\left\langle r_{0}\right\rangle$ is the initial average droplet diameter of the emulsion. Considering "reasonable" numerical values, namely $\sigma=10^{-2} \mathrm{~N} \cdot \mathrm{m}^{-1}, \mathrm{~V}_{\mathrm{m}}=10^{-3} \mathrm{~m}^{3} \cdot \mathrm{mol}^{-1}$, $\left\langle\mathrm{r}_{0}\right\rangle=0.5 \mu \mathrm{m}$, the molar fraction of insoluble oil $x_{i, \min }$ has a value of $1.6 \%$ at $\mathrm{T}=298 \mathrm{~K}$.

In practice, the main factor determining the rate of OR is the water solubility of the oil phase, $C(\infty)$. This is why, OR is not usually a concern for emulsions based on oils with very low water solubility such as long chain triglycerides. Instead, OR may occur rapidly for fine emulsions $(<\mathrm{r}><<1 \mu \mathrm{m})$ prepared using oils with a significant watersolubility like short chain triglycerides, essential oils, etc. (Wooster et al., 2008). In this latter case, OR can be arrested by dissolving for instance a long chain triglyceride within the oil phase prior to the emulsification at a mole fraction given by eq. 5 .

\section{Interdroplet interactions in the presence of protein-polysaccharide mixtures}

Proteins and polysaccharides play a key role in the stabilization of food emulsions. Mixtures of both biopolymers can be found in the ingredients of a wide range of products like mayonnaise or ice cream. Proteins are primarily used as emulsifying agents. Polysaccharides are used to inhibit gravity-induced creaming and further phase separation in W/O emulsions during long-term quiescent storage. The main effect of polysaccharides is based on the viscosity increase of the aqueous continuous phase located in the gaps between the dispersed droplets. This is certainly a realistic explanation at low oil volume fractions, where the welldispersed droplets can be separately immobilized in an entangled polysaccharide network, and the buoyancy force acting on each droplet is insufficient to overcome the yield stress of the surrounding gel. For moderately concentrated emulsions (say 30 vol. \% oil), this explanation has to be revised because the evolving microstructure of the emulsion is highly heterogeneous (Moschakis et al., 2006). Indeed, in concentrated O/ $\mathrm{W}$ emulsions, the mechanism by which hydrocolloid thickeners control creaming phenomena involves the interconnected depletion-flocculated network of oil droplets.

The overall stability and texture of food emulsions containing biopolymer mixtures depends not only on the intrinsic properties of the individual ingredients, but also on the nature and strength of the protein-polysaccharide interactions. In some cases, protein and polysaccharide interactions may synergistically improve the overall emulsion stability. When attractive forces arise between them, complexation results and the protein-polysaccharide complexes that may adsorb at the oil/water interface and efficiently stabilize oil-in-water emulsions (Neirynck et al., 2007). When protein-stabilized emulsions are subjected to heat treatment, for pasteurization or sterilization purposes, they generally tend to aggregate because of protein denaturation and bridging and the resulting emulsions become flocculated. However, incubation of proteinpolysaccharide mixtures at high temperature may result in covalently linked amphiphilic conjugates whose adsorption at the droplet surfaces enhances the repulsive steric interactions. The resulting emulsions exhibit no sign of droplet aggregation after prolonged heating and remain stable against creaming (Diftis and Kiosseoglou, 2006).

\section{Bulk rheological properties of concentrated emulsions}

Since emulsions are dispersions of deformable droplets, they can span droplet volume fractions from zero to almost one. Much effort has been spent in studying the rheology of diluted emulsions. At low volume fraction, emulsions consist of spherical droplets and such systems flow easily and exhibit shearthinning behavior. A significant change in the rheological behaviour is observed if the droplets are concentrated up to volume fractions higher than the fraction $\phi^{*}$ corresponding to the random close packing of hard spheres. For randomly packed monodisperse spheres, $\phi^{*}=0.64$. Above $\phi^{*}$, the droplets can no longer pack without deforming (figure 2); although being composed of fluids only, emulsions resemble an elastic solid as they do not flow under they own weight. The elasticity arises because the repulsive droplets have been concentrated up to a sufficiently large volume fraction that permits the storage of interfacial energy (Princen, 1986; Princen and Kiss, 1986). Two compressed droplets will begin to deform before their interfaces actually touch, due to the intrinsic repulsive interactions between them. Emulsions minimize their total free energy by reducing the repulsion, which may have different origins, at the expense of creating some additional surface area by stretching the droplet. The excess surface area of the droplets determines the equilibrium elastic energy stored by the interfaces. When a small sinusoidal shear stress, $\tau$, is applied to an emulsion, the resulting strain, $\gamma$, is proportional to $\tau$, with $\tau=\mathrm{G} \gamma$. The shear modulus, $\mathrm{G}$, is determined by the additional excess surface area created by shear deformation. This latter is frequency-dependent and can be considered as the sum of two 


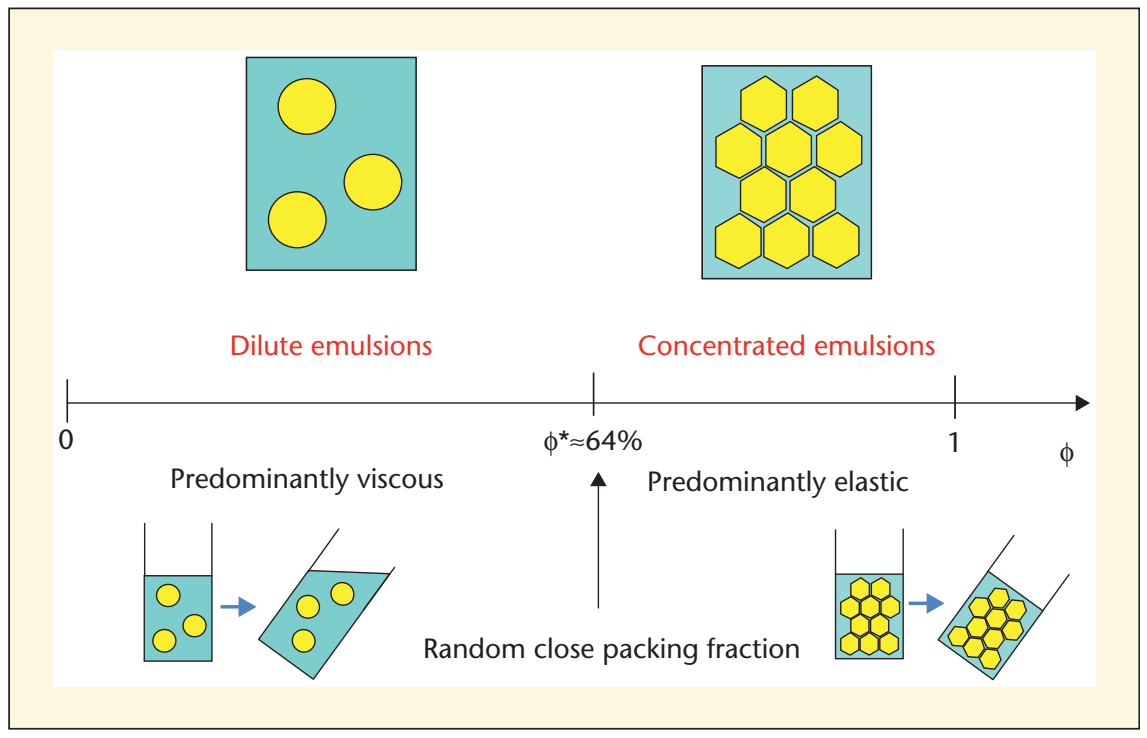

Figure 2. Evolution of the rheological properties in monodisperse emulsions as a function of the droplet volume fraction, $\phi$.

contributions. The in-phase contribution, $\mathrm{G}^{\prime}$, is linked to the stored energy (solid-like behavior), while the $\pi / 2$ outof-phase contribution, $\mathrm{G}^{\prime \prime}$, is related to the dissipated energy (fluid-like behavior). Both moduli are increasing functions of the droplet fraction. In concentrated emulsions, the elastic modulus is significantly higher than the loss modulus, reflecting the essentially solid nature of the materials. Interestingly, when normalized by half the Laplace pressure $(\sigma /<r)>)$, the rescaled data of $G^{\prime}$ and $G^{\prime \prime}$ all fall onto single master curves, whatever the average droplet size of the emulsions as shown in figure 3 (Mason et al., 1995). The scaling with Laplace pressure confirms that the origin of elasticity results from the storage of energy due to the stretching of the interfaces. Moreover, the scaling with the droplet fraction indicates that the elasticity of these compressed droplets depends only on the packing geometry.

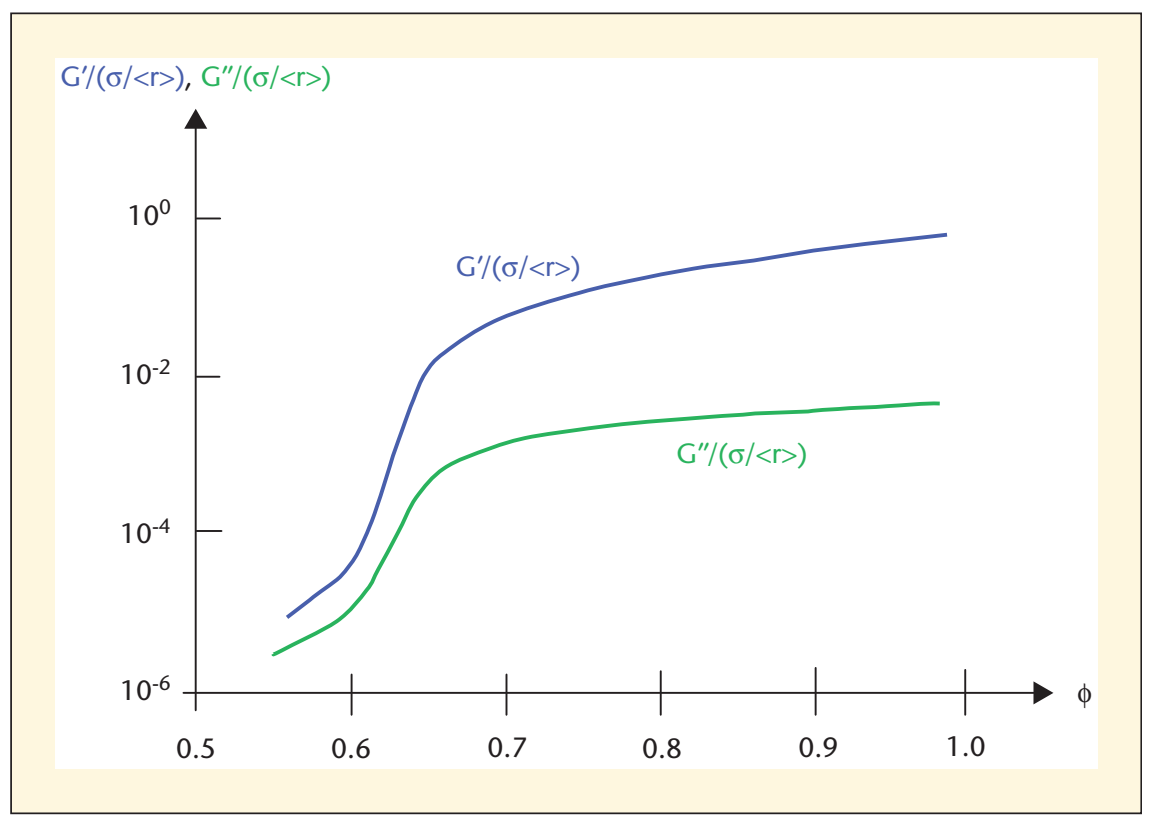

Figure 3. Evolution of the shear moduli $G^{\prime}$ and $G^{\prime \prime}$, normalized by Laplace pressure, as a function of the droplet volume fraction, $\phi$, for monodisperse emulsions.
Proteins adsorbed at the oil-water interface are tightly packed and may experience strong lateral attractive interactions. They form bi-dimensional layers at the interface with intrinsic 2D viscoelastic properties. As a consequence, droplet deformation is controlled not only by Laplace pressure but also by the 2D interfacial viscoelastic properties. This is why the dimensionless shear elastic modulus $\mathrm{G}^{\prime} /(\sigma /<\mathrm{r}>$ ) of protein-stabilized emulsions is generally larger than for lowmolecular-weight surfactant-stabilized emulsions (Dimitrova et al., 2001; Bressy et al., 2003; Derkatch et al., 2007).

\section{Emulsions based on crystallizable oils}

Very often the dispersed phase in emulsions is composed of totally or partially crystallized oils. For instance, this is the case of dairy emulsions. Upon cooling, the spherical and smooth surface of the warm dispersed droplets which is controlled by surface tension evolves into a rough and rippled surface due to the formation of irregularly shaped/oriented crystals. In such conditions, the droplets are prone to the so-called "partial coalescence" phenomenon (Boode and Walstra, 1993). Crystals located nearby the oil/water interface can protrude into the continuous phase and when such crystals are present within the thin film separating two droplets, they may pierce it and bridge the surfaces, causing the droplets to coalesce (figure 4). If the crystallized fraction within the globules is sufficient, the intrinsic firmness of the fat network hinders relaxation to the spherical shape driven by surface tension after each coalescence event. As time passes, large clusters appear and grow by the accretion of any other primary droplet or cluster until a rigid network made of partially coalesced droplets is formed, in which the original droplet shape remains recognizable.

Large surface-active species like proteins are highly efficient in stabilizing partially crystallized droplets against partial coalescence. The emulsions then exhibit long-term kinetic stability in quiescent storage conditions and can even be submitted to high shear forces without being destroyed. Instead, surfactants with short molecular chain length generally do not provide a sufficient degree of stabilization and the emulsions may become lumpy a few hours after their 


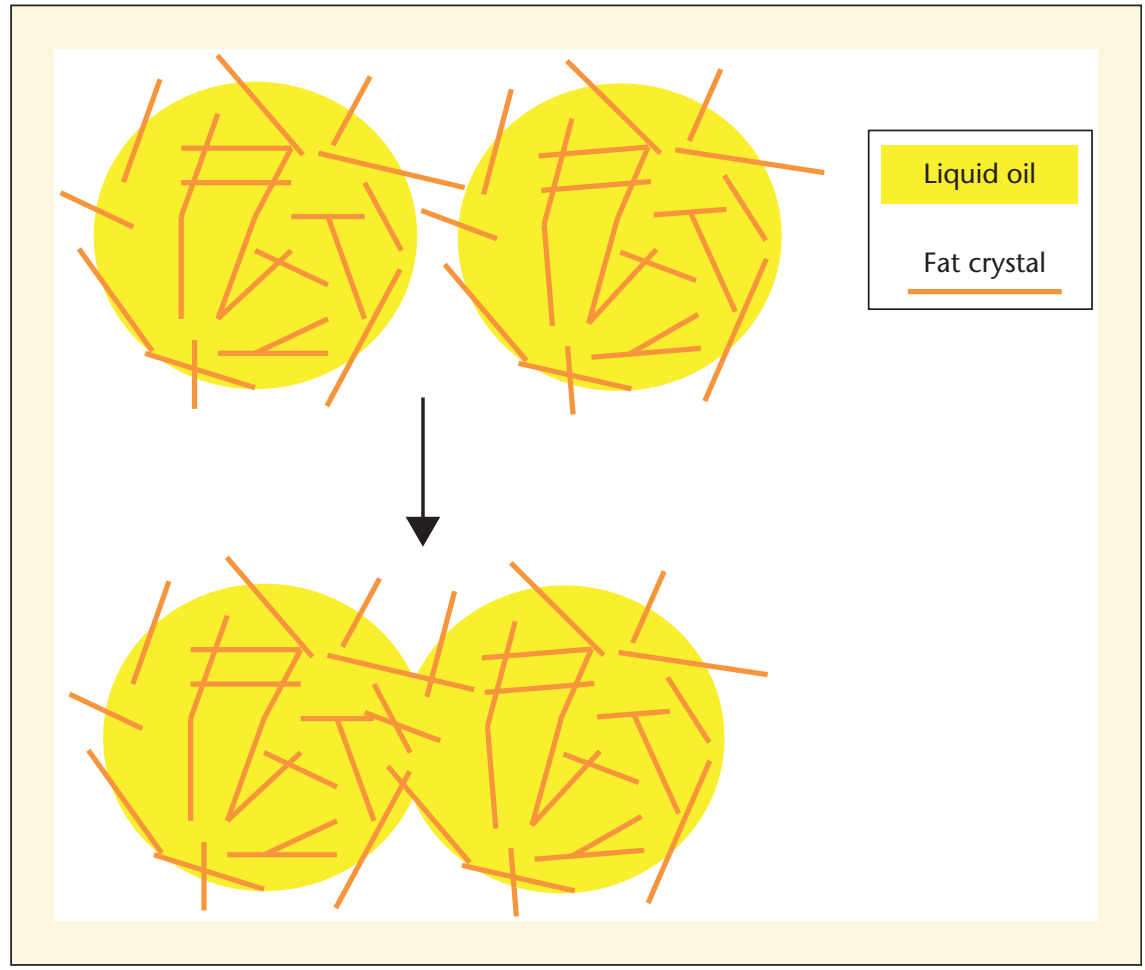

Figure 4. Scheme of the partial coalescence process. Fat crystals protruding from the interface pierce the film in between two adjacent droplets. Shape relaxation is inhibited because of the intrinsic firmness of the solid fat network.

preparation. Addition of small amounts of surfactant in protein-stabilized emulsions is a common way to monitor the sensitivity towards partial coalescence (Thivilliers et al., 2008; Fredrick et al., 2010). The displacement of proteins from the oil interface by added surfactants (Mackie et al., 1999) contributes to emulsion instability and induces sensitivity to partial coalescence.

\section{Thermally induced partial coalescence}

Partial coalescence may be provoked in quiescent conditions upon application of a specific thermal treatment called "Tempering". Tempering consists of applying a temperature cycle that produces partial melting of the crystal mass (warming step), followed by recrystallization (cooling step). Tempering is known as an efficient way to transform an initially fluid cream into a material with thick or solid consistency (Boode et al., 1991). Thivilliers et al. (2006) observed a significant increase in the emulsion firmness in triglyceride (anhydrous milk fat and cocoa butter)-in-water emulsions stabilized by sodium caseinate/ surfactant mixtures. Owing to tem- sions turned into hard gels that became trapped into a deep metastable state as roughness (flow hindering). they could be stored for several weeks without any appreciable change. The gel structure formed after a long interval of tempering was a continuous network of partially aggregated fat droplets. The progressive increase of the bulk elastic modulus $G^{\prime}$ during tempering was interpreted within the frame of percolation theory. The results suggest that partial coalescence involves irreversible bridging between a crystallized patch in one droplet surface and a melted patch on an adjacent droplet surface (figure 4). Consequently, the rate of partial coalescence reaches a maximum value for a well-defined solid fraction within the oil phase. It was also shown that the coalescence rate and thus the gelling kinetics were controlled by the oil solid fraction, the average droplet size and the surfactant-to-protein molar ratio.

Gelling in emulsions has also been studied in model crystallisable droplets made of alkanes or paraffin oils with high melting temperature. Following the pioneering work of Golemanov et al. (2006), Kahn et al. (2007) have investigated two possible routes to produce emulsion gels in quiescent conditions, both of them exploiting the crystallized state of the droplets (figure 5). One is due to partial coalescence which induces formation of a rigid network of interconnected droplets. This scenario occurs

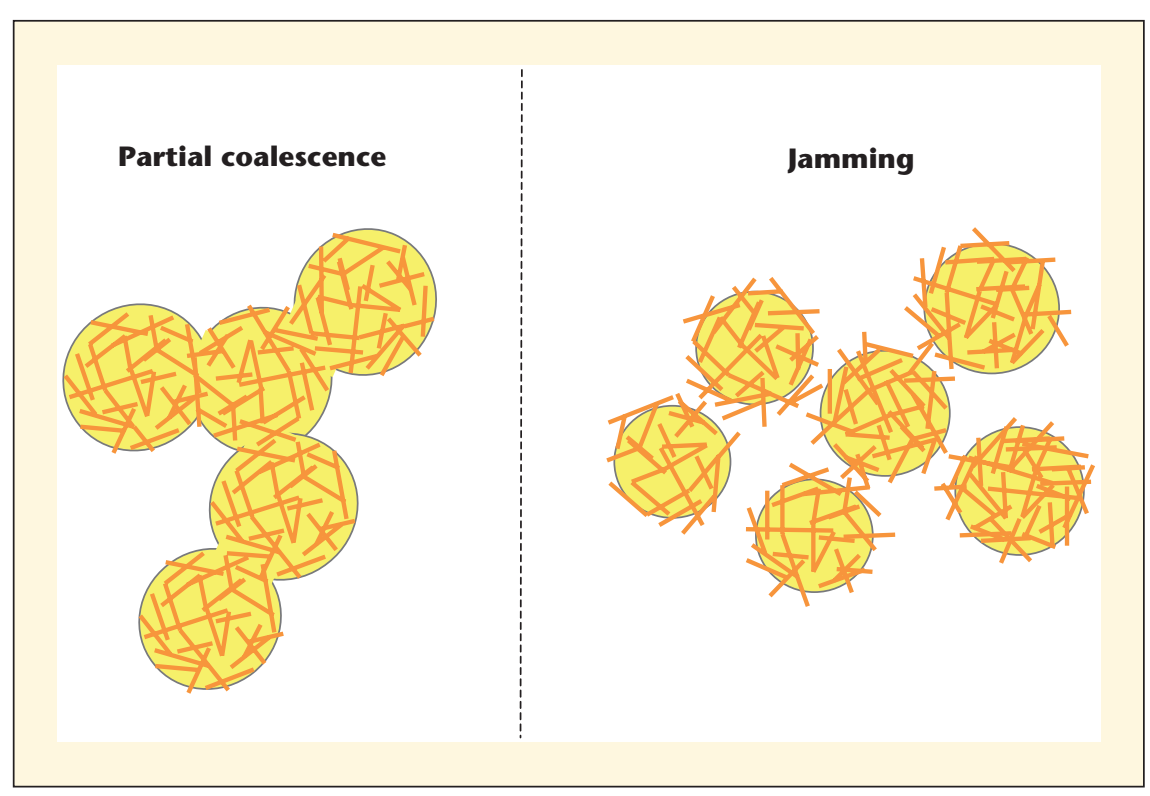

Figure 5. Two possible configurations for gelled materials based on crystallizable droplets: partial coalescence and jamming. When partial coalescence occurs, the elasticity arises from the network of interconnected droplets, whereas in the jamming process the elasticity is due to the fact that the individual droplets cannot slide one against each other because of surface 
preferentially when the average droplet size is large. The second route occurs without film rupturing (no coalescence) and is provoked by a restriction of the internal dynamics ("jamming") due to surface roughness. This latter situation is preferentially observed in emulsions with small average droplet diameter.

\section{Shear-induced partial coalescence}

Shear forces due to processing may induce dramatic and irreversible changes in the structure of materials comprising semi-solid droplets. For instance, the fabrication of viscoelastic aerated foods like whipped creams or ice creams is based on the application of intense mechanical agitation which promotes the formation of an interconnected droplets network (van Aken, 2001; Drelon et al., 2006). Shear-induced partial coalescence is also exploited to separate fat from the aqueous phase of native dairy creams in order to obtain butter. Many cosmetic products are oilin-water emulsions containing semi-crystalline waxes and the texture of such materials is controlled by the instabilities occurring under shear. The evolution scenario depends on the formulation, temperature, and shear history of the sample.

Many papers in the literature have reported destabilization of emulsions made of partially and/or fully crystallized

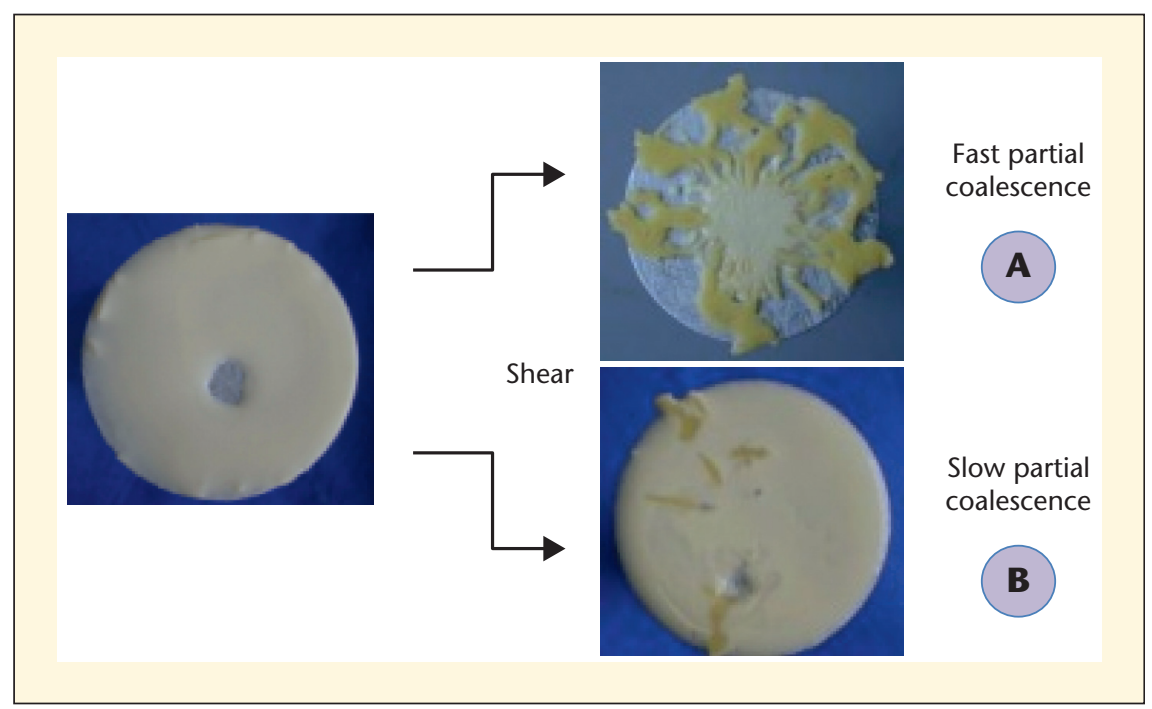

Figure 6. Two possible pathways for the shear-induced evolution in emulsions based on crystallizable oils: (A) formation of a gel that spans over the whole volume and (B) nucleation/ growth of randomly distributed clumps (adapted from Thivilliers et al., 2010).

droplets submitted to shear (Hinrichs and Kessler, 1997; Davies et al., 2000; Xu et al., 2005; Guery et al., 2006). Thivilliers-Arvis et al. (2010) provided insight into how semi-solid oil-in-water emulsions may become destabilized by partial coalescence when subjected to moderate shear flows. They designed observation methods to visualize oilcontinuous regions that result from coalescence of droplets. Quantitative relations between the composition of the droplets (in terms of solid fraction) and the parameters that characterize the instability onset (magnitude, duration or frequency of the applied strain) were obtained. With these tools, they identified two mechanisms for the propagation of coalescence through the network of partially coalesced droplets. The evolution of the primary emulsions according one of them is mainly determined by the rate of partial coalescence. Emulsions prone to fast coalescence undergo gelling before massive macroscopic clumping occurs. Instead, in emulsions exhibiting low coalescence rates, randomly distributed clumps are formed and grow over time (figure 6). The coalescence rate and thus the destabilization pathway depend on the crystal size and/or protrusion at the oil/ water interface, both properties being controlled by the average droplet size and by the protein-to-surfactant molar ratio. 
in a dramatic reduction in triglyceride absorption, in part because the network of fat crystals provided the agglomerates with an internal scaffold to resist redispersion as they passed through the pylorus (region of the stomach that connects to the small intestine).

\section{Conclusion}

Emulsions are out-of-equilibrium materials governed not only by thermodynamic conditions but also by the entire process history. The basic knowledge about the physicochemical properties and kinetic evolution of emulsions is progressing but the inherent complexity of such materials is permanently raising novel fundamental questions at the frontier between physics, chemistry and biology. Many emulsions based on lipids in food, cosmetic and pharmaceutical industries are designed to interact with human body. The behavior of emulsions once ingested, spread on skin or introduced in the bloodstream trough parenteral administration is far to be fully understood and consolidating knowledge in that field would certainly improve their performance (in terms of therapeutic, nutritional, organoleptic, properties). Controlling the fate of emulsions in body is certainly one the most challenging issues of emulsion science within the next years or decades.

\section{REFERENCES}

Armand M. Lipases and lipolysis in the human digestive tract: where do we stand? Curr Opin Clin Nutr Metab Care 2007; 10: 156-64.

Armand M, Pasquier B, Borel $\mathrm{P}$, et al. Emulsion and absorption of lipids: the importance of physicochemical properties. OCL 1997; 4: 178-85.

Aronson MP. The role of free surfactant in destabilizing Oil-in-Water emulsions. Langmuir 1989; 5: 494-501.

Asakura S, Oosawa J. Interaction between particles suspended in solutions of macromolecules. J Polym Sci 1958; 32: 183-92.

Bibette I, Roux D, Nallet F. Depletion interactions and fluid-solid equilibrium in emulsions. Phys Rev Lett 1990; 65: 2470-3.

Bressy L, Hebraud P, Schmitt V, Bibette J. Rheology of emulsions stabilized by solid interfaces. Langmuir 2003; 19: 598-604.

Boode K, Bisperink C, Walstra P. Destabilization of $\mathrm{O} / \mathrm{W}$ emulsions containing fat crystals by temperature cycling. Colloids and Surfaces A 1991; 61: 55-74.
Boode K, Walstra P. Partial coalescence in oil-in-water emulsions. 1: Nature of the aggregation. Colloids and Surfaces A 1993; 81: 121-37.

Carriere F, Barrowman JA, Verger R, Laugier $R$. Secretion and contribution to lipolysis of gastric and pancreatic lipases during a test meal in humans. Gastroenterology 1993; 105: 876-88.

Carrière F, Grandval P, Gregory PC, Renou C, Henniges F, Sander-Struckmeier S, Laugier R. Does the pancreas really produce much more lipase than required for fat digestion? J Pancreas 2005; 6: 206-15.

Davies E, Dickinson E, Bee R. Shear stability of sodium caseinate emulsions containing monoglyceride and triglyceride crystals. Food Hydrocolloids 2000; 14: 145-53.

Hinrichs ], Kessler H. Fat content of milk and cream and effects on fat globule stability. J Food Sci 1997; 62: 992-5.

Dickinson E, Golding M, Povey MJW. Creaming and Flocculation of Oil-in-Water emulsions containing sodium caseinate. / Colloid Interface Sci 1997; 185: 515-29.

Derkatch SR, Levachov SM, Kuhkushkina AN, Novossyolova NV, Kharlov AE, Matveenko VN. Rheological properties of concentrated emulsions stabilized by globular protein in the presence of non-ionic surfactant. Colloids Surfaces A 2007; 298: 225-34

Diftis N, Kiosseoglou V. Stability against heatinduced aggregation of emulsions prepared with a dry-heated soy protein isolate-dextran mixture. Food Hydrocolloids 2006; 20: 787-92.

Dimitrova T, Leal Calderon F. Bulk elasticity of concentrated protein-stabilized emulsions. Langmuir 2001; 17: 3235-44.

Dimitrova TD, Leal-Calderon F, Gurkov TD, Campbell B. Surface forces in model Oil-inWater emulsions stabilized by proteins. $A d v$ Colloid Interface Sci 2004; 73: 108-9.

Drelon N, Gravier E, Boisserie L, Omari A, Leal-Calderon $F$. Influence of temperature cycling ("tempering") on the mechanical properties of whipped dairy creams. Int Dairy / 2006; 16: 1454-63.

Fave G, Coste TC, Armand M. Physicochemical properties of lipids: new strategies to manage fatty acid bioavailability. Cell Mol Biol 2004 ; 50: 815-31.

Feinle C, Christen M, Grundy D, Faas $H$, Meier O, Otto B, Fried M. Effects of duodenal fat, protein or mixed-nutrient infusions on epigastric sensations during sustained gastric distension in healthy humans. Neurogastroenterol Motil 2002; 14: 205-13.

Fredrick E, Walstra P, Dewettinck K. Factors governing partial coalescence in oil-in-water emulsions. Adv Colloid Interface Sci 2010; 153: 30-42.
Golding M, Wooster TJ, Day L, Xu M, Lundin L, Keogh J, Clifton P. Impact of gastric structuring on the lipolysis of emulsified lipids. Soft matter 2011; 7: 3513-23.

Golding M, Wooster TJ. The influence of emulsion structure and stability on lipid digestion. Curr Opin Colloid Interface SCi 2010; 15: 90-101.

Golemanov K, Tcholakova S, Denkov ND, Gurko T. Selection of surfactants for stable paraffin-in-water dispersions, undergoing solid-liquid transition of the dispersed particles. Langmuir 2006; 22: 3560-9.

Guery J, Bertrand E, Rouzeau C, Levitz P, Weitz DA, Bibette J. Irreversible Shear-Activated Aggregation in Non-Brownian Suspensions. Phys Rev Lett 2006; 96: 198361.

Higuchi WI, Misra J. Physical degradation of emulsions via the molecular diffusion route and the possible prevention thereof. J Pharm Sci 1962; 51: 459-66.

Israelachvili JN. Intermolecular and Surface Forces (with applications to colloidal and biological systems). Academic Press, London, Orlando, San Diego, New York, Toronto, Montreal, Sydney, Tokyo, 1985.

Kabalnov A. Ostwald ripening and related phenomena. J Dispersion Sci Tech 2001; 22: 1-12.

Kabalnov AS, Wennerström H. Macroemulsion stability: the oriented wedge theory revisited. Langmuir 1996; 12: 276-92.

Kahn J, Schmitt V, Drelon N, Thivilliers F, LealCalderon F. Gelling of oil-in-water emulsions comprising crystallized droplets. Langmuir 2007; 23: 4792-9.

Leal-Calderon F, Schmitt V, Bibette J. Emulsion Science. Basic Principles. $2^{\text {nd }}$ version. Springer, New York, 2007.

Lifshitz IM, Slyozov VV. The kinetics of precipitation from supersaturated solid solutions. J Phys Chem Solids 1961; 19: 35-50.

Lundin L, Golding M, Wooster TJ. Understanding food structure and function in developing food for appetite control. Nutr Diet 2008; 65: S79-85.

Mabille C, Schmitt V, Gorria P, Leal-Calderon $F$, Faye $V$, Deminière $B$, Bibette J. Rheological and shearing conditions for the preparation of monodisperse emulsions. Langmuir 2000; 16: 422-9.

Mackie AR, Gunning AP, Wilde PJ, Morris VJ. The orogenic displacement of protein from the air/water interface by surfactant. / Colloid Interface Sci 1999; 210: 157-66.

Mason TM, Bibette J, Weitz DA. Elasticity of compressed emulsions. Phys Rev Lett 1995; 75: 2051-4

Mattes RD. The role of macronutrients in appetite regulation. Can J Diet Pract Res 2007; 68: 2-Insert Sum 2007. 
McClements DJ. Edible nanoemulsions: fabrication, properties, and functional performance. Soft Matter 2011; 7: 2297-316.

Meller A, Stavans J. Stability of emulsions with nonadsorbing polymers. Langmuir 1996; 12: 301-4.

Moschakis T, Murray BS, Dickinson E. Particle tracking using confocal microscope to probe the micro rheology in a phaseseparating emulsion containing nonadsorbing polysaccharide. Langmuir 2006; 22: 4710-9.

Neirynck N, van der Meeren P, LukaszewiczLausecker M, Cocquyt J, Verbeken D, Dewettinck K. Influence of $\mathrm{pH}$ and biopolymer ratio on whey protein-pectin interactions in aqueous solutions and in O/W emulsions. Colloids Surfaces A 2007; 298: 99-107.

Princen HM. Osmotic pressure of foams and highly concentrated emulsions. 1. Theoretical considerations. Langmuir 1986; 2: 519-24.
Princen HM, Kiss AD. Rheology of foams and highly concentrated emulsions: III. Static shear modulus. J Colloid Interface Sci 1986; 112:427-37.

Schmitt V, Cattelet C, Leal-Calderon F. Coarsening of alkane-in-water emulsions stabilized by non-ionic poly (ethylene oxide) surfactants: the role of molecular permeation and coalescence. Langmuir 2004; 20: 46-52.

Shah P, Bhalodia D, Shelat P. Nanoemulsion: a pharmaceutical review. Sys Rev Pharm 2010; 1: 24-32.

Thivilliers F, Drelon N, Schmitt V, LealCalderon F. Bicontinuous emulsion gels induced by partial coalescence: kinetics and mechanism. Europhys Lett 2006; 76: 332-8.

Thivilliers F, Laurichesse E, Saadaoui H, LealCalderon F, Schmitt V. Thermally-induced gelation of Oil-in-Water Emulsions comprising partially crystallized droplets: the impact of interfacial oil crystals. Langmuir 2008; 24 : 13364-75.

Thivilliers-Arvis F, Laurichesse E, Schmitt V, Leal-Calderon F. Shear-induced gelling and clumping in Oil-in-Water emulsions comprising partially crystallized droplets. Langmuir 2010; 26: 16782-90.

van Aken GA. Aeration of emulsions by whipping. Colloids Surfaces A 2001; 190: 333-354.

Wagner C. Theorie der alterung von niederschlägen durch umlösen (Ostwald Reifung). Z Elektrochem 1961; 65: 581-91.

Wooster TJ, Golding M, Sanguansri P. Impact of oil type on nanoemulsion formation and Ostwald ripening stability. Langmuir 2008; 24: 12758-65.

Xu W, Nikolov A, Wasan D. Shear-induced fat particle structure variation and the stability of food emulsions: I. Effects of shear history, shear rate and temperature. J Food Eng 2005; 66: 97-105. 